\title{
Classification of Ball Mill Acoustic for Predictive Grinding using PCA on Time and Frequency Domain Data
}

\author{
Sonali Sen, Arup Kumar Bhaumik, Jaya Sil
}

\begin{abstract}
The process of comminution is nondeterministic in nature, so deriving out a designated size range on crushing by fixing the parameters of the mill is not possible in mining industry. Loss of materials in huge amount is an obvious phenomenon due to under sizing of materials in transit. The aim of the paper is to predict the state of grinding and the particle size distribution (psd) during any desired stage of crushing in the ball mill. The acoustic sensors have been used to capture audio signals at different running conditions of the ball mill and analyzed to develop the prediction model. In the proposed work first Genetic Algorithm (GA) based predictive procedure is applied on the fragmented signal to extract the parameters of genetic operators and store them in a table. We also apply Gaussian Mixture Model (GMM) to obtain the psd of each fragment and Fuzzy C-means (FCM) clustering algorithm is employed to classify the distributed signal. The psd of each fragment has been stored in another table. The experiment is conducted for different raw materials with different size distribution. At run time the material grinding procedure is operated and stopped automatically based on the trained controlled parameters corresponding to the desired stage of grinding. The psd of experimental output is verified with the desired psd obtained during training and stored in the table. The proposed method exhibits significant improvement in prediction performance and outcomes are verified with the experimental results.
\end{abstract}

Keywords : Acoustic Signature, Ball Mill, Crossover and Mutation, Fuzzy C means, Gaussian Mixture Model

\section{INTRODUCTION}

$\mathrm{I}_{\mathrm{n}}$ Mining Industry, particle grinding is required to obtain desired particle size with different smaller size ranges, used for different purposes. However, the existing process is performed manually since installation of sensors e.g. optical, magnetic or temperature in the mill is not practicable. In the existing systems [1][2], dozers are used to prepare the input mix (feed) with different particle sizes. Each dozer corresponds to a particular size range of particles and by

Revised Manuscript Received on February 07, 2020.

* Correspondence Author

Sonali Sen*, Department of Computer Science, St. Xavier's College, Kolkata, India. Email: sonalisen@sxccal.edu

Arup Kumar Bhaumik, Department of Computer Science, RCCIIT, Kolkata, India. Email: arup_bhaumik@yahoo.com

Jaya Sil, Department of Computer Science, IIEST, Kolkata, India. Email: jayaiiests@gmail.com

(C) The Authors. Published by Blue Eyes Intelligence Engineering and Sciences Publication (BEIESP). This is an open access article under the CC BY-NC-ND license (http://creativecommons.org/licenses/by-nc-nd/4.0/) varying the speed of the dozer motor the amount of dozing of each range has been controlled. On preparation of the input mix in a vessel of the mill, the iron balls are inserted as grinding media and thereafter the mill motor is allowed to start to crush the particles for some specified time (i.e. particle residence time) after that the mill is stopped manually. The crushed materials after removing the iron balls are fed into a stack of screen for classification of particles in different size ranges. The desired particle size ranges are collected from the classifier while others are fed into the respective input stack to prepare the input mix for the next stage of grinding. In such operation, the undersized materials are lost, which is huge in case of manually controlled machines. Thus, the stack of screen or classifier used in tandem with the mill seems to provide a closed loop condition. Moreover, the process is purely off-line where the exact state of breakage of particle is unpredictable since each particle breaks in different manner. Researchers suggested several mathematical relationships such as Rosin-Rammler, Gaudin-Schuman, Benette-Colleman [3] equations, which to some extent can forecast how the broken particles vary with different size range. These equations have been tested in early part of our simulation work [4] but none of them found good enough to interpret results at coarse or fine particle size range. Moreover, the existing equations are not suitable enough to fit in an autonomous system where control can be imparted using information transaction only without considering the operational conditions of grinding [5]. Similar work has been published in [6][7] describing a scheme to use the acoustic signature to regulate the mill and the product in off-line mode of operations.

In this work, we consider the ball mill as our experimental grinding machine and propose a technique to predict the psd at any desired stage of grinding. In this proposed approach we first train the system using acoustic signal recorded during crushing operation of different materials with variable sizes and different operational conditions (with load and empty) of the mill. The recorded signal is then fragmented equivalent to equal time interval and filtered. We apply crossover, selection and mutation operators of Genetic Algorithm (GA) on the fragmented signal to simulate information exchange and diversity in psd while crashing the particles. Crossover operation is performed on each two consecutive acoustic fragmented signal and the off-springs are mutated.

Off-springs are evaluated using the proposed fitness function and the selected one predicts the next segment at a particular stage of grinding. For each segment parameters of genetic operators are stored in a table. 
Also Gaussian Mixture Model (GMM) is applied on the fragmented signal and we obtain the psd of the raw material at each stage of crushing. To classify the distribution Fuzzy C-means (FCM) clustering algorithm is chosen since the distribution is overlapping due to additive nature of different signals. The simulated result is verified with the actual screened raw material showing equivalent distribution and the corresponding psd is stored in a table. At runtime proposed method predicts the current breakage stage and size distribution of the test particles.

The paper has been divided into 4 sections. In Section 2 the proposed method is described while in section 3 the results are presented. Finally, in section 4 the conclusion is discussed. and to monitor the mill operation in real time, respectively. The experimentation method of capturing the acoustic signal of a ball mill at empty state and while at the running state in various loading conditions are described below. A schematic diagram of the proposed approach is shown in figure1.

\section{A. Preprocessing}

Microphones are fitted outside the mill and we capture the audio signal in its empty and load varying conditions with different raw materials and size distribution. The experiment was conducted in a University Mining Laboratory so that we carefully avoided all the unwanted noise. Also we pass the captured sound through a band pass filter by knowing the frequency range [4][10][14] of the mill in its empty and load varying conditions. The output is stored in wav [7] format. Figure 2 shows the plotting of continuous signal of the mill in its loaded condition.

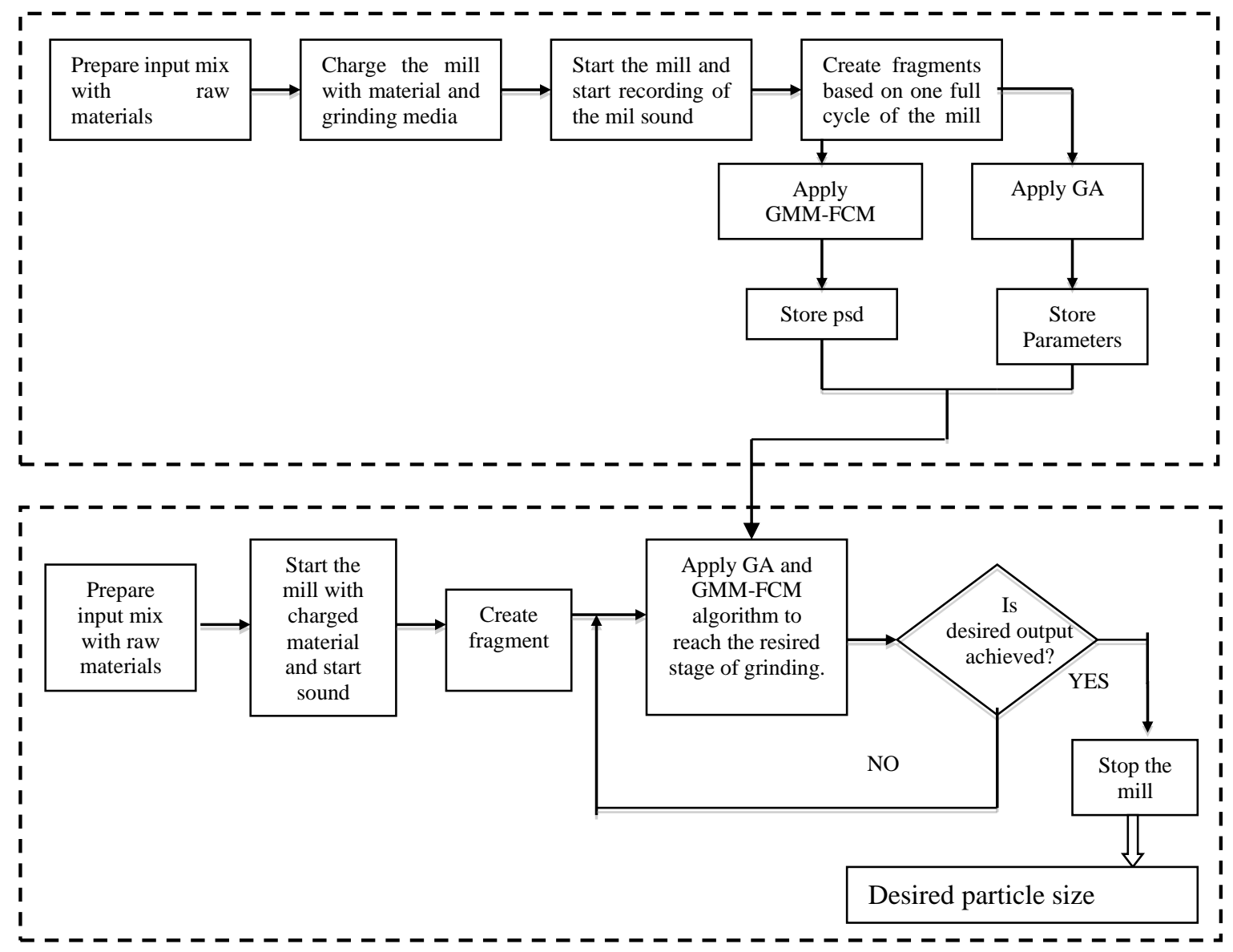

Fig. 1. Block diagram with training and testing phase of the

\section{PROPOSED APPROACH}

The experiments have been carried out in two modes - off-line and on-line for the preparation of knowledge base training set 


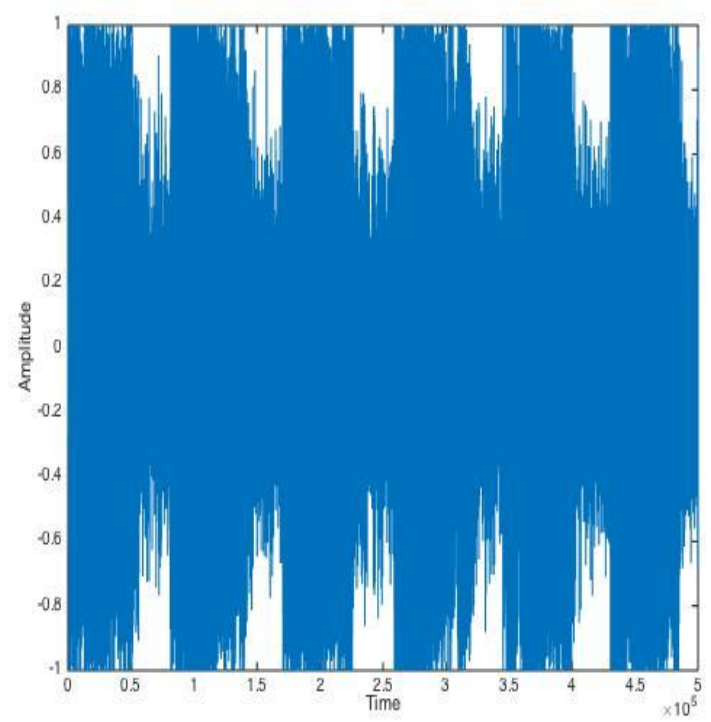

Fig. 2. Mill sound recorded in continuous basis

\section{B. Fragmentation}

The recording of the acoustic signal is performed until the material was grinded according to the required psd. Since the sound was captured for a long time during grinding, a voluminous data (in terms of billions) has been generated; analysis of which for predicting test signal in real time becomes a challenge. We first analyze the experimental signal and observed that the acoustics of the ball mill is gradually changing while approaching towards its fine crushing. During the experiment we noticed that the ball mill completed its one full cycle within a unit time for different types of materials with different psd. Therefore, to achieve scalability, signal obtained due to one full rotation of the mill is fragmented of equal length. From the fragmented signals feature vectors are generated consisting of time domain data (amplitude value). The plot of two consecutive fragments in its load varying conditions is shown in figure 3.
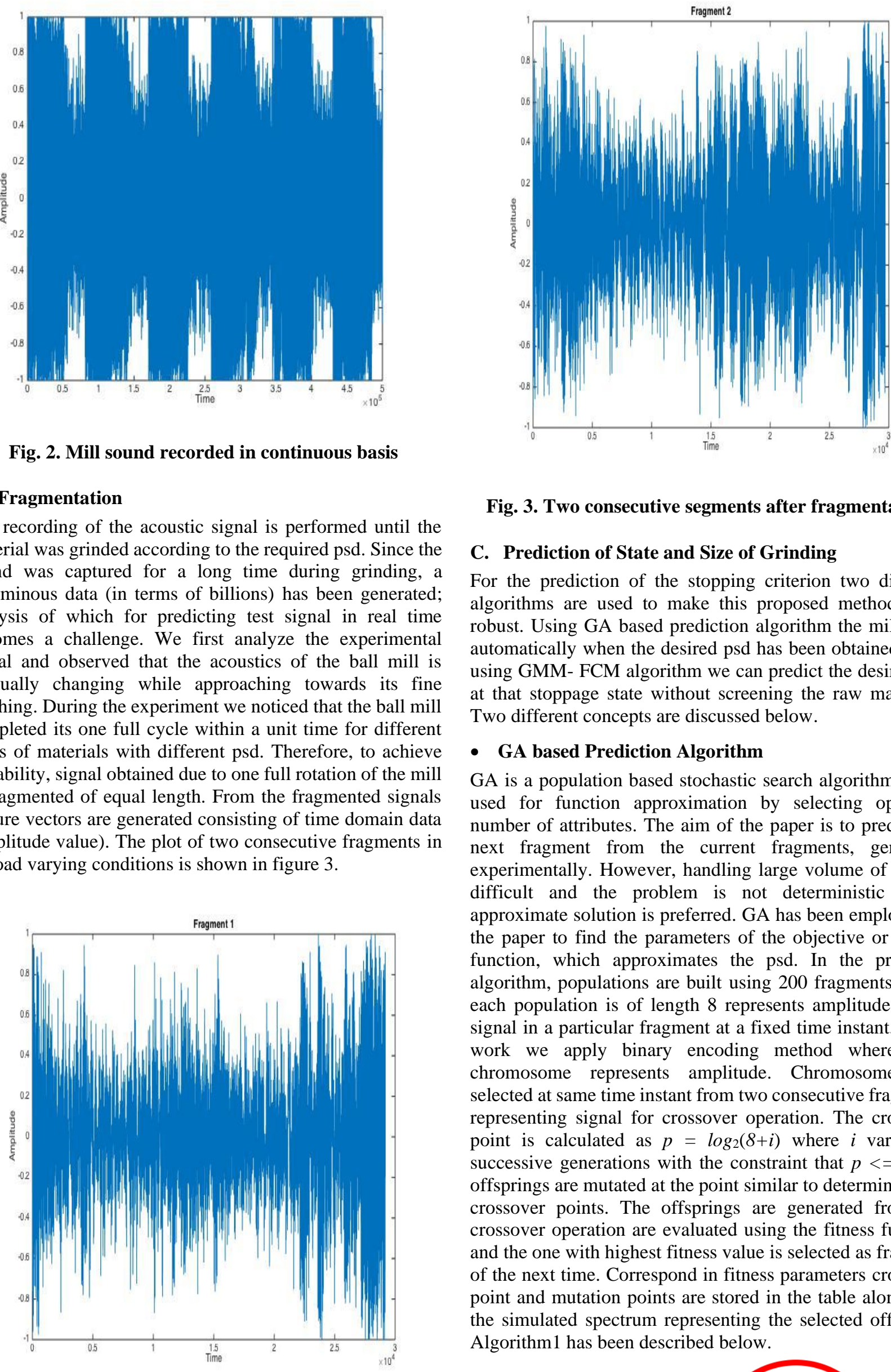

Fig. 3. Two consecutive segments after fragmentation

\section{Prediction of State and Size of Grinding}

For the prediction of the stopping criterion two different algorithms are used to make this proposed method more robust. Using GA based prediction algorithm the mill stops automatically when the desired psd has been obtained. Also using GMM- FCM algorithm we can predict the desired psd at that stoppage state without screening the raw materials. Two different concepts are discussed below.

\section{- GA based Prediction Algorithm}

GA is a population based stochastic search algorithm [8][9] used for function approximation by selecting optimum number of attributes. The aim of the paper is to predict the next fragment from the current fragments, generated experimentally. However, handling large volume of data is difficult and the problem is not deterministic rather approximate solution is preferred. GA has been employed in the paper to find the parameters of the objective or fitness function, which approximates the psd. In the proposed algorithm, populations are built using 200 fragments where each population is of length 8 represents amplitude of the signal in a particular fragment at a fixed time instant. In the work we apply binary encoding method where each chromosome represents amplitude. Chromosomes are selected at same time instant from two consecutive fragments representing signal for crossover operation. The crossover point is calculated as $p=\log _{2}(8+i)$ where $i$ varies for successive generations with the constraint that $p<=8$. The offsprings are mutated at the point similar to determining the crossover points. The offsprings are generated from the crossover operation are evaluated using the fitness function and the one with highest fitness value is selected as fragment of the next time. Correspond in fitness parameters crossover point and mutation points are stored in the table along with the simulated spectrum representing the selected offspring. Algorithm1 has been described below.

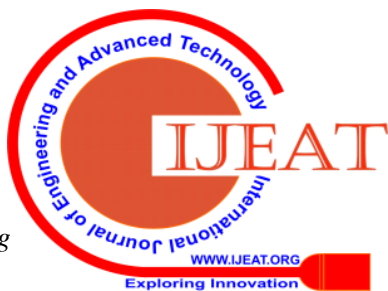




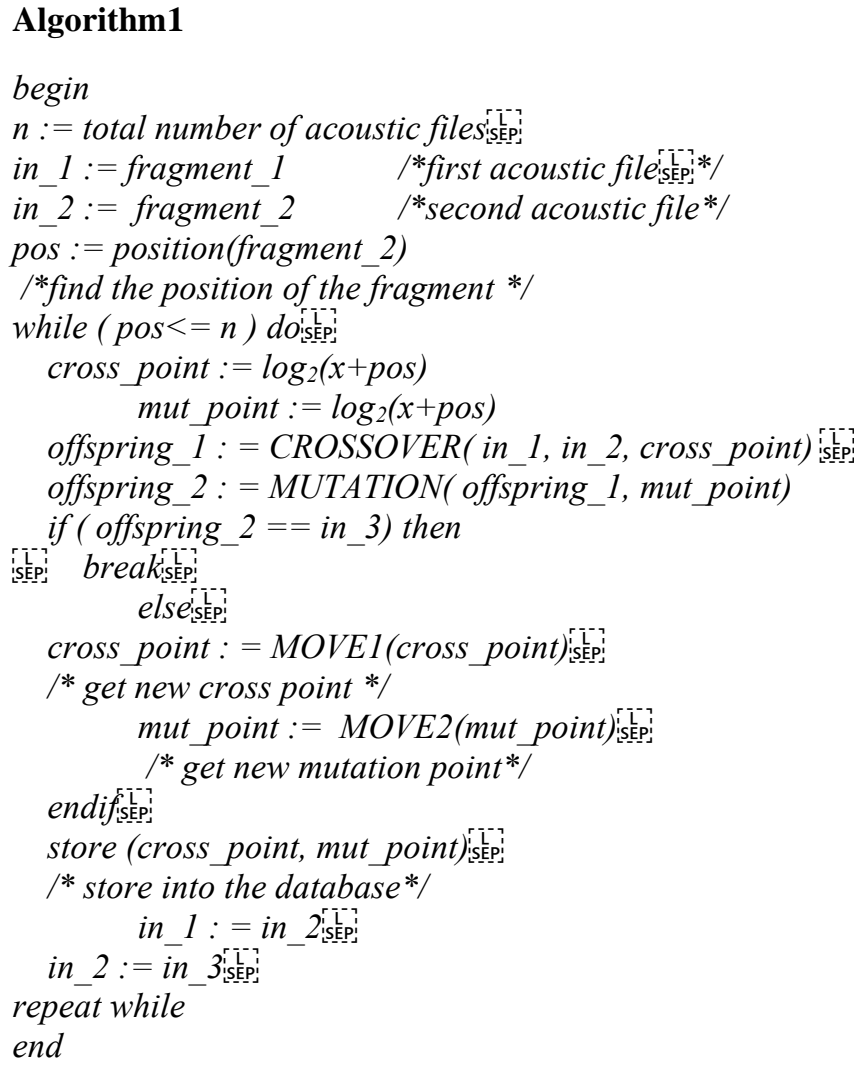

The fitness function is defined by equation (1).

$$
y=1 / e^{-\alpha x}
$$

where, $\alpha$ is a constant, $x$ is the variable representing amplitude of the signal. Appropriate value of $\alpha$ is obtained after training while considering absolute difference between the simulated and the experimental fragments.

Using binary encoding, any two chosen chromosomes can be represented as a string of 0 s and $1 \mathrm{~s}$ as:

Chromosome 1: 10110010

Chromosome 2: 11000001

After applying 1-point crossover off-Springs are

Offspring1: 10110001

Offspring2: 11000010

Flip the value of the chosen gene as 0 to 1 and 1 to 0 which produce the mutated off-springs as follows :

Offspring1: 10100011

Offspring2: 11001010

Then we select one of the off-spring which is fitted best.

\section{- GMM-FCM Algorithm}

In this part of work, we take the fragmented signal as input and try to extract the information about the particle size distribution. The sound is a mixture of the mill, grinding media and all the different size particles rolled inside the mill. We know GMM is a distribution technique of a mixture of $K$ separate multivariate normal distributions, where each with own parameters represented as whole $\theta$. The probability density function of GMM with given parameters is defined by equation (2).

$$
p(x \mid \theta)=\sum_{k=1}^{K} \alpha_{k} \mathcal{N}\left(x_{i} \mid \mu_{k}, \Sigma_{k}\right),
$$

where $x_{i}$ is a $D$ dimensional point, $\mathcal{N}$ denotes the normal probability distribution of an observation, and $\alpha_{k}, \mu_{k}$ and $\Sigma_{k}$ are the amplitude, mean and covariance matrix of each Gaussian matrix. There is no analytic solution to estimate the parameters of a mixture of Gaussians from a sample data set. The standard approach is to iteratively maximize the likelihood function of the mixture model, called Expectation Maximization (EM) algorithm. GMM sometimes described as soft clustering method. The posterior probabilities for each point indicate that each data point has some probability of belonging to each cluster. So we applied FCM because we know that the distribution may have overlapping characteristics. Based on the distribution information of GMM, we apply FCM clustering algorithm to find the exact number of clusters from the acoustic signal. This algorithm works by assigning membership to each data point corresponding to each cluster center on the basis of distance between the cluster center and the data point. More the data is nearre to the cluster center more is its membership towards the particular cluster. Clearly, summation of membership of each data point should be equal to one. After each iteration, membership and cluster centers are updated using equations (3) and (4).

$$
\mu_{i j}=\frac{1}{\sum_{k=1}^{c} d_{i j} / d_{j k}\left(\frac{2}{m-1}\right)}
$$

$v_{j}=$
$\left.\left(\sum_{i=1}^{n}\left(\mu_{i j}\right)^{m} x_{i}\right) / \sum_{i=1}^{n}\left(\mu_{i j}\right)^{m}\right), \forall_{j}=1,2, \ldots \ldots \ldots, c$

where, ' $n$ ' is the number of data points.

' $v_{j}$ ' represents the $j^{\text {th }}$ cluster center.

' $m$ ' is the fuzziness index $\mathrm{m} \epsilon[1, \infty]$.

' $C$ ' represents the number of cluster center.

' $\mu_{i j}{ }^{\prime}$ 'represents the membership of $i^{\text {th }}$ data to $j^{\text {th }}$ cluster center.

' $d_{i j}$ ' represents the Euclidean distance between $i^{\text {th }}$ data and $j^{\text {th }}$ cluster center.

Main objective of FCM algorithm is to minimize $\mathrm{J}(\mathrm{U}, \mathrm{V})$ given in equation (5).

$$
J(U, V)=\sum_{i=1}^{n} \sum_{j=1}^{c}\left(\mu_{i j}\right)^{m} \| x_{i}-\left.v_{j}\right|^{2}
$$

Where, ' ||$x_{i}-v_{j} \mid$ ' is the Euclidean distance between $i^{\text {th }}$ data and $j^{\text {th }}$ cluster center. 
In this part of work we try to predict the desired particle size distribution of the material. At the beginning we prepare the input mix and take a particular size distribution of the raw material. During crushing, particle size distribution changes over time. Using GMM-FCM algorithm we try to predict that different size distribution at each stage of grinding. Let $X=$ $\left\{x_{1}, x_{2}, x_{3} \ldots, x_{n}\right\}$ be the set of data points at any stage and $V=$ $\left\{v_{1}, v_{2}, v_{3}, \ldots, v_{c}\right\}$ be the set of cluster centers. Then we apply the following algorithm:

\section{Algorithm 2}

Step 1: Randomly select ' $C$ ' cluster centers.

Step 2: Calculate the fuzzy membership ' $\mu_{i j}$ ' using:

$$
\mu_{i j}=\frac{1}{\sum_{k=1}^{c} d_{i j} / d_{i k}^{\left(\frac{2}{m-1}\right)}}
$$

Step 3: Compute the fuzzy centers ' $v_{j}$ ' using :

$\left.v_{j}=\left(\sum_{i=1}^{n}\left(\mu_{i j}\right)^{m} x_{i}\right) / \sum_{i=1}^{n}\left(\mu_{i j}\right)^{m}\right), \forall_{j}=1,2, \ldots \ldots \ldots, c$

Step 4 : Repeat steps 2 and 3 until the minimum ' $j$ ' value is achieved or $\|U(k+1)-U(k)\|<\beta$,

where, ' $k$ ' is the iterations,

' $\beta$ ' is the termination criterion between $[0,1]$,

' $U=\left(\mu_{i j}\right) n^{*} c$ ' is the fuzzy membership matrix.

Information about the cluster is stored in the table for testing. At run time, we start a mill with a particular raw material and with a fixed psd. A real time recording is also started while the system starts generating the fragments. Whenever the system starts crushing and we isolate two initial fragments for testing. Then the proposed prediction algorithm is applied on the first two fragments. A priori information is also stored about the required psd. During the testing phase the system predicts the next fragment based on crossover point and mutation point stored in the table. The proposed model calculates at what time or how many different mill cycles, the system needs to be stopped. Accordingly a counter will be started and whenever it will reach to its desired count the system is stopped. The system also gives the information about the psd of the current state.

\section{RESULTS}

A database for crushing of iron ore with a priori information about the particle size distribution is shown in table 1 . The table consists of the segments, simulated output, crossover point and mutation point. The comparison is done in between the simulated output with the experimental output. The comparison was performed using two different methods - (1) Fitness function with $\alpha=0.57$ and (2) Euclidian distance. The analysis of the simulated output for load varying conditions of the ball mill has been done using the fitness function and Euclidean distance. Using Euclidean distance we measure that the simulated results are $75 \%$

similar with the experimental one. It implies that the simulated output follows the experimental results.

Figure 4 shows the inputs - fragment 1 and fragment 2, simulated output and experimental output. Figure 5 shows the comparison between simulated and experimental output for three different sets of inputs. From the diagram it has been observed that there is a very negligible difference supplementing the performance of our algorithm. We calculate L2 - norm to find the error between the simulated and experimental results for large number of data set. The average error is order of 0.07 .

Table 1 : Experimental Inputs and Simulated Parameters

\begin{tabular}{|c|c|c|c|c|c|}
\hline $\begin{array}{c}\text { Experime } \\
\text { ntal } \\
\text { fragment } \\
1\end{array}$ & $\begin{array}{c}\text { Experim } \\
\text { ental } \\
\text { fragmen } \\
t 2\end{array}$ & $\begin{array}{c}\text { Simulat } \\
\text { ed } \\
\text { Output }\end{array}$ & $\begin{array}{l}\text { Cross } \\
\text { over } \\
\text { Point }\end{array}$ & $\begin{array}{c}\text { Mutat } \\
\text { ion } \\
\text { point }\end{array}$ & $\begin{array}{c}\text { Estimated } \\
\text { Error } \\
\text { Between } \\
\text { Experimental } \\
\text { and Simulated } \\
\text { results }\end{array}$ \\
\hline$F 1$ & $F 2$ & S3 & 4 & 5 & 0.077 \\
\hline$F 2$ & F3 & S4 & 4 & 5 & 0.08 \\
\hline F3 & F4 & $S 5$ & 4 & 6 & 0.067 \\
\hline F4 & $F 5$ & S6 & 9 & 5 & 0.09 \\
\hline$F 5$ & F6 & S7 & 8 & 4 & 0.065 \\
\hline$F 6$ & F7 & $S 8$ & 4 & 4 & 0.067 \\
\hline F7 & $F 8$ & S9 & 5 & 5 & 0.07 \\
\hline$F 8$ & F9 & $S 10$ & 4 & 4 & 0.066 \\
\hline F9 & $F 10$ & $S 11$ & 4 & 4 & 0.056 \\
\hline$F 10$ & $F 11$ & $S 12$ & 6 & 6 & 0.067 \\
\hline$F 11$ & $F 12$ & $S 13$ & 4 & 5 & 0.071 \\
\hline$F 12$ & $F 13$ & $S 14$ & 4 & 5 & 0.065 \\
\hline$F 13$ & $F 14$ & S15 & 4 & 5 & 0.046 \\
\hline$F 14$ & $F 15$ & $S 16$ & 4 & 5 & 0.06 \\
\hline$F 15$ & $F 16$ & $S 17$ & 4 & 4 & 0.066 \\
\hline$F 16$ & $F 17$ & S18 & 4 & 4 & 0.07 \\
\hline$F 17$ & $F 18$ & S19 & 4 & 4 & 0.065 \\
\hline$F 18$ & $F 19$ & $S 20$ & 7 & 5 & 0.063 \\
\hline$F 19$ & $F 20$ & $S 21$ & 5 & 4 & 0.063 \\
\hline$F 20$ & $F 21$ & $S 22$ & 5 & 4 & 0.067 \\
\hline$F 21$ & $F 22$ & $S 23$ & 8 & 9 & 0.072 \\
\hline$F 22$ & $F 23$ & $S 24$ & 7 & 4 & 0.071 \\
\hline$F 23$ & $F 24$ & $S 25$ & 12 & 7 & 0.076 \\
\hline$F 24$ & $F 25$ & $S 26$ & 4 & 4 & 0.07 \\
\hline$F 25$ & $F 26$ & $S 27$ & 7 & 5 & 0.06 \\
\hline$F 26$ & $F 27$ & $S 28$ & 4 & 4 & 0.07 \\
\hline$F 27$ & $F 28$ & $S 29$ & 4 & 4 & 0.066 \\
\hline$F 28$ & $F 29$ & S30 & 5 & 4 & 0.069 \\
\hline$F 29$ & F30 & S31 & 4 & 5 & 0.066 \\
\hline F30 & F31 & S32 & 8 & 5 & 0.065 \\
\hline
\end{tabular}



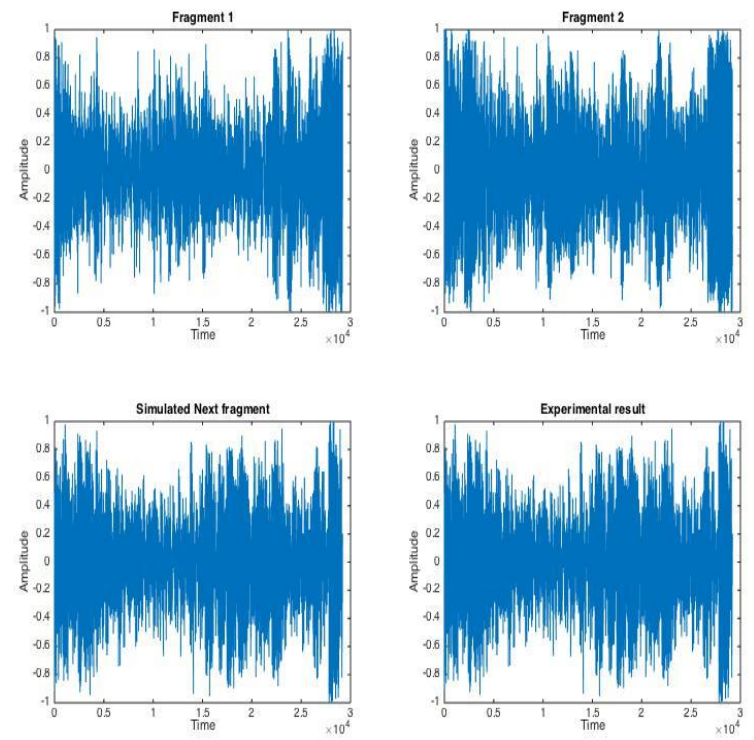

Fig. 4. Result set 1 using the proposed algorithm

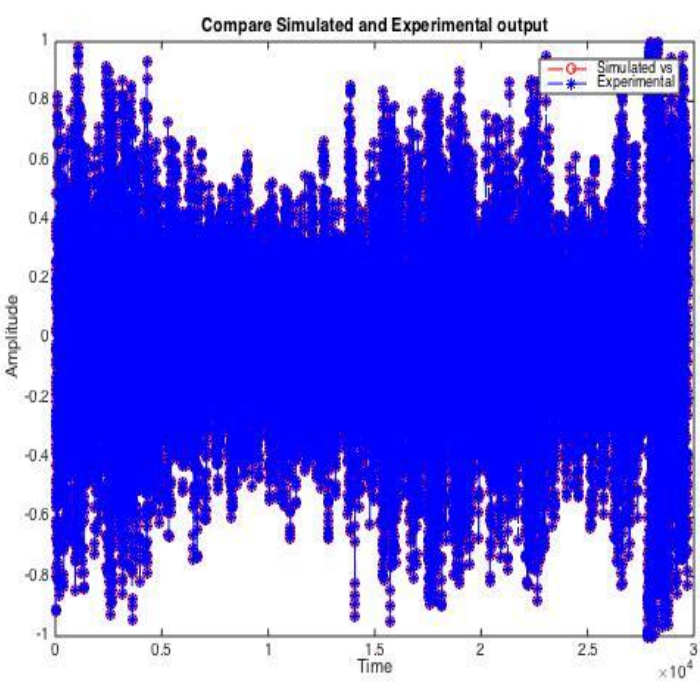

Fig. 5(a) Comparison between the simulated and experimental result set 1

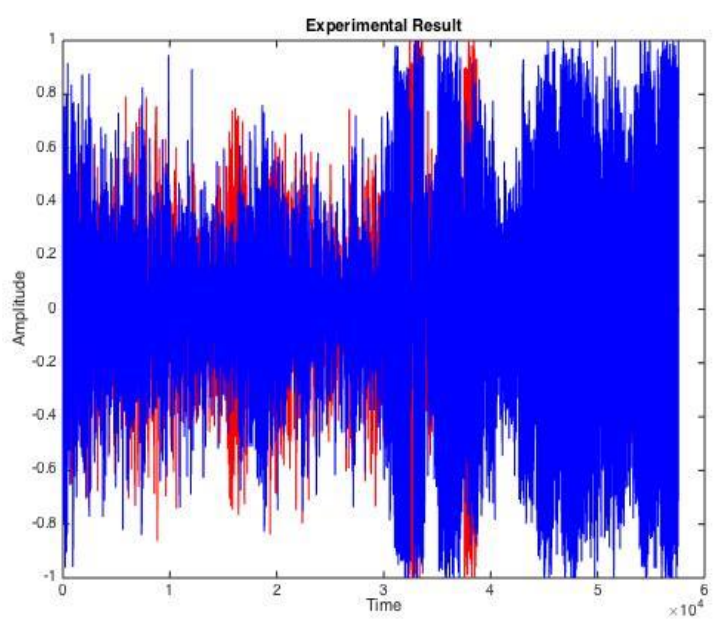

Fig. 5(b) Comparison between the simulated and experimental result set 2

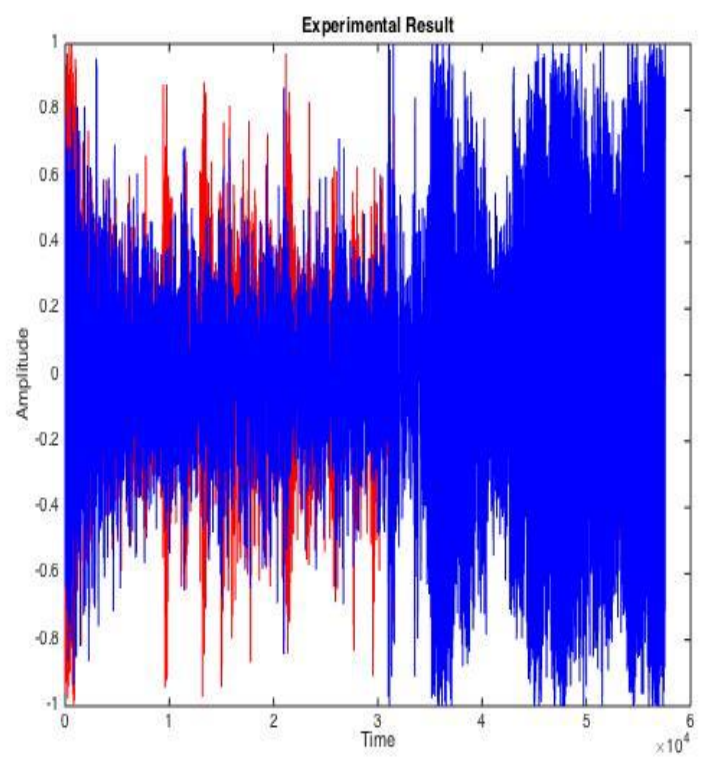

Fig. 5(c) Comparison between the simulated and experimental result set 3

At the beginning we need to prepare the input mix. In our experiment we consider 6 different size particles as input mix. Figure 6 shows the particle size distribution with the corresponding cluster plot of that distributed data. There are 6 clusters, 4 for different particle sizes and 2 for mill and grinding media.
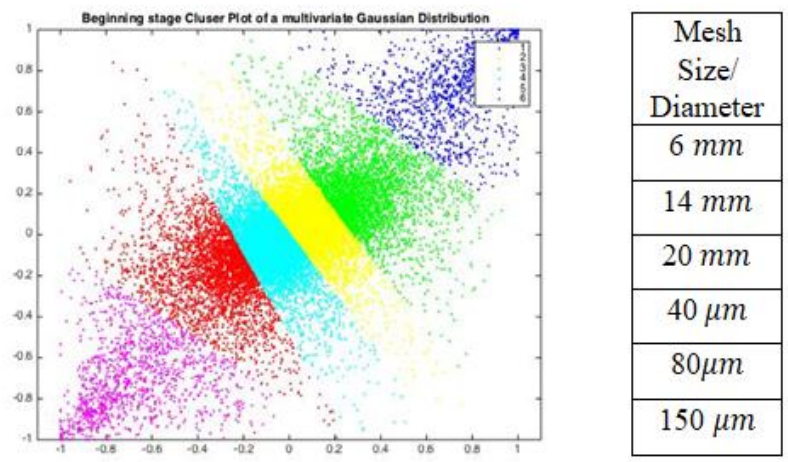

Fig. 6. Cluster plot at the beginning of crushing

Gradually when the crushing operation is carried away, the particles are grinded and the size distributions increases. After 2 minutes of crushing if we screen the input mix and simulate the cluster plot of the distributed data we obtain the output provided in figure 7.

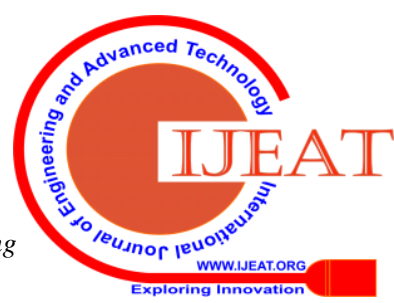




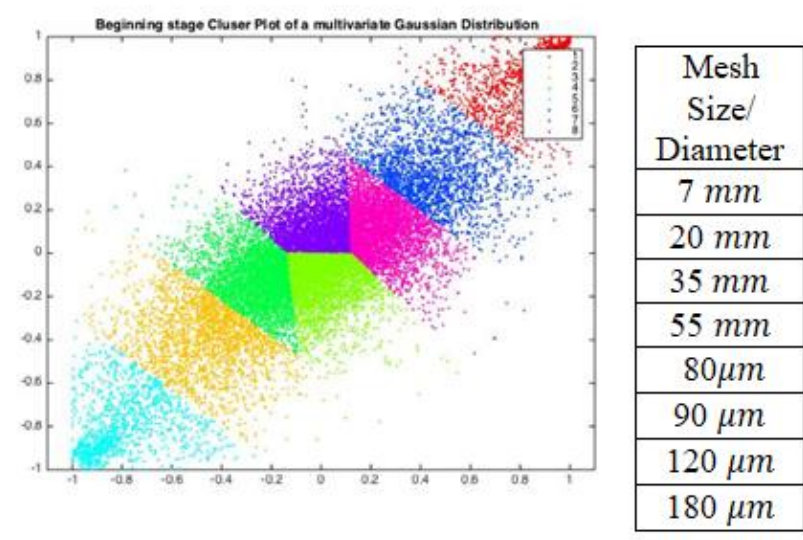

Fig. 7. Different particle sizes and Cluster plot after 2 minutes of crushing

At the end of crushing when we screen the input mix and simulate the cluster plot of the distributed data we obtain the output as shown in figure 8 .

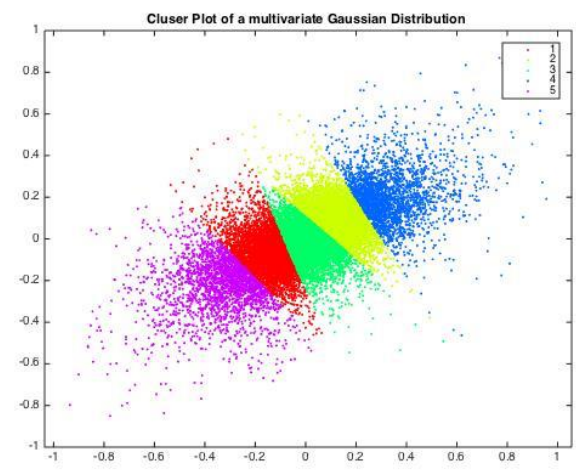

\begin{tabular}{|c|}
\hline $\begin{array}{c}\text { Mesh } \\
\text { Size/ } \\
\text { Diameter }\end{array}$ \\
\hline $20 \mathrm{~mm}$ \\
\hline $80 \mathrm{~mm}$ \\
\hline $100 \mu \mathrm{m}$ \\
\hline $200 \mu \mathrm{m}$ \\
\hline $220 \mu \mathrm{m}$ \\
\hline
\end{tabular}

Fig. 8 Different particle sizes and Cluster plot at the end of crushing

After each 2 minutes of crushing till the end we screen the material to check the distribution of particle sizes. Also we simulate the program to find the distribution and plot the clusters. Experimental output is compared with the simulated one.

\section{CONCLUSION}

In this paper we propose a method for real time mill operation by predicting the particle size distribution of raw materials during crushing. The simulated results correlate with the experimental results. Several methods towards prediction of grinding have been proposed in our earlier work $[10][11][12][14]$. The experiment is conducted using different crushing materials. In each different case, the real time testing satisfies the experimental output. It has been concluded that the operators of GA used to find the stopping condition of the mill based on the knowledge base is effective for real time operation. The intelligent real time control system is proposed in this paper allow closed loop grinding without any material loss. Acoustic evolution is an integral part of the work. Its importance basically lies with the formation of the knowledge base of the method and generation of feedback signal that is the key factor in close loop information system. The model is not only valid for the ball mill but also expected to work well with other different kind of reactors/crushers/compound machines. Under different training conditions convergence of error in acoustic signature has been investigated and provides satisfactory results.

\section{REFERENCES}

1. W.A. Gills, "Mineral Processing”, Springer Verlag, Germany, 1991.

2. H.Taggert, "Hand book of mineral processing", SME publishers, 1951. ĩ

3. B. A. Wills, "Mineral Processing Technology", Pergamon Press, U.K., 1986. 'ín:

4. A. Bhaumik, J. Sil, and S. Banerjee, "Hybrid Control of Tumbling Mill using NN based Schematics", International Journal of Information and Computing (IJICS) vol.2, no. 1, pp. - 21-28, June 1999. 'SLEP:

5. A. Bhaumik, J. Sil, S. Maity and T. Das, "Designing an Intelligent expert Control System using Acoustic Signature for Grinding Mill operation", International Conference on Industrial Technology (ICIT 2006), IEEE Catalog Number 06TH8924C, ISBN 1-4244-0726-5, Library of Congress, 2006935034, 2006.

6. A. Bhaumik, J. Sil, and S. Banerjee, "A New Supervised Training Algorithm For Generalized Learning “, Third International Conference on Computational Intelligence and Multimedia Applications, ICCIMA '99, pp. 489-493, 1999.

7. Ze-Nian Li, Mark S. Drew, Jiangchuan Liu, "Fundamentals of Multimedia", Second Edition, ISSN 1868-0941, ISBN 978-3-31905289-2, pp -164 -165.

8. Andrew R. Webb, Keith D. Copsey, "Statistical Pattern Recognition", Third Edition by Mathematics and Data Analysis Consultancy,

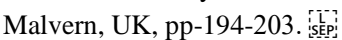

9. C. M. Bishop, "Pattern Recognition and Machine Learning”, Springer, USA, pp 1-30. in

10. A. Bhaumik, J. Sil, and S. Banerjee, "Designing of Intelligent Hybrid Control System for Tumbling Mill Operation", Proceedings of the 6th International Conference on Information Technology (CIT2003), Allied Publishers Pvt. Ltd., pp 246-249, Bhubaneswar, India, 2003. is [-p?

11. A. Bhaumik, J. Sil, and S. Banerjee: "Designing of Intelligent Expert Control System Using Petri Net for Grinding Mill Operation”, WSEAS Transactions on Information Science and Applications, Issue 4, Volume 2, pp. 360-365, ISSN 1790-0832, April 2005. is îp?

12. S. Sen and A. Bhaumik, "Design of Intelligent Control System Using Acoustic Parameters for Grinding Mill Operation", National Conference on Advancement of Computing in Engineering Research, pp. 261-268, 2013. DOI : 10.5121/csit.2013.3224. [istep.

13. Alan P Schmidt and Trevor K. M. Stone, "Music classification and identification system", Tech. Rep., Department of Computer Science, University of Colorado, Boulder, 2002. [iَ

14. Sonali Sen, A. Bhaumik, J. Sil, 'Mathematical Modeling of Predictive Grinding for Ball Mill', IEEE TENCON, 2016, pp. 1184-1188, DOI : 978-1-5090-25978/16

\section{AUTHORS PROFILE}

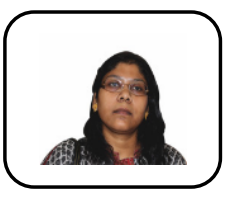

Sonali Sen, received her M.Sc. degree in Computer Science from the University of Calcutta in the year 2003 and done her M.Tech in Computer Science and Engineering from the University of Calcutta in the year 2005. She is pursuing her Ph.D on Signal Processing from West Bengal University of Technology. She is working as Assistant Professor (Sr.) in the department of Computer Science, St. Xavier's College, Kolkata from 2006. She has published more than 15 journal and conference papers. Her research interests include Signal Processing, Graph Theory, Security, applied cryptography and Image Processing. 


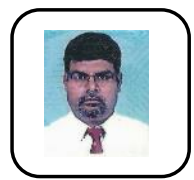

Prof.(Dr.) Arup Kr. Bhaumik, Principal, RCCIIT did B.Tech \& M.Tech in Computer Sc. \& Engg. From C.U. and $\mathrm{PhD}$ in Computer Technology from BESU, Member IEEE, Life Member ISTE, FOSET \& IEI Patent holder on Acoustic Controller for Grinding Mill, Served academics in different Capacities from Lecturer, Asst. Professor, Professor to Principal in Different Organizations starting from NERIST ( Govt. of India), Haldia Institute of Technology, MCET, Gurunanak Institute of Technology, RCCIIT. Published several Papers in reputed Journals \& conference proceedings and books are also written by him.

Prof. (Dr.) Jaya Sil, did her B.E, M.E, Ph. D in Computer Science. She is the professor in department of Computer Science at IIEST, Shibpur. She has published papers in IEEE Transactions as well as different international Journals. Her area of interests are Image Processing, Pattern Recognition, Multi Agent systems and Bio-Informatics. 